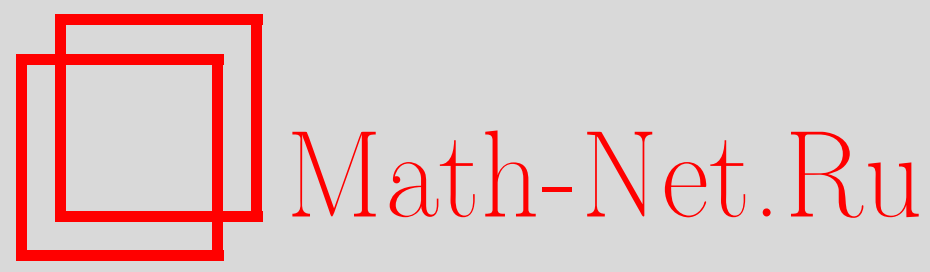

А. А. Шкаликов, Д.-Г. Бак, Мультипликаторы в дуальных соболевских пространствах и операторы Шрёдингера с потенциалами-распределениями, Матем. заметки, 2002, том 71, выпуск 5, 643-651

DOI: https://doi.org/10.4213/mzm373

Использование Общероссийского математического портала Math-Net.Ru подразумевает, что вы прочитали и согласны с пользовательским соглашением http://www. mathnet.ru/rus/agreement

Параметры загрузки:

IP : 44.207 .124 .84

26 апреля 2023 г., 08:25:39

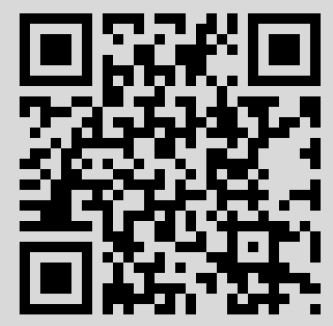




\title{
МУЛЬТИПЛИКАТОРЫ В ДУАЛЬНЫХ СОБОЛЕВСКИХ ПРОСТРАНСТВАХ И ОПЕРАТОРЫ ШРЁДИНГЕРА С ПОТЕНЦИАЛАМИ-РАСПРЕДЕЛЕНИЯМИ
}

\author{
Дж.-Г. Бак, А. А. Шкаликов
}

В работе получены достачные условия принадлежности функций-распределений пространству мультипликаторов из соболевского пространства $H_{p}^{\alpha}\left(\mathbb{R}^{n}\right)$ в дуальное пространство $H_{p^{\prime}}^{-\alpha}\left(\mathbb{R}^{n}\right)$. При $\alpha>n / p$ найден критерий, т.е. дано полное описание рассматриваемого пространства мультипикаторов. Полученные результаты применяются для определения операторов Шрёдингера с потенциалами-распределениями.

Библиограффия: 8 названий.

Целью этой статьи является изучение мультипликаторов из соболевского пространства $H_{p}^{\alpha}\left(\mathbb{R}^{n}\right)$ в дуальное пространство $H_{p^{\prime}}^{-\alpha}\left(\mathbb{R}^{n}\right)$, где $\alpha \geqslant 0, p \geqslant 1$ и $p, p^{\prime}-$ сопряженные по Гёльдеру числа, т.е. $1 / p+1 / p^{\prime}=1$.

Мультипликаторам в соболевских пространствах с неотрицательньми индексами посвящена обширная литература. Подробности можно найти в книге Мазьи и Шапошниковой [1]. В недавней работе Нейман-заде и Шкаликова [2] было показано, что мультипликаторы из пространства Соболева $H_{2}^{\alpha}\left(\mathbb{R}^{n}\right)$ в $H_{2}^{-\alpha}\left(\mathbb{R}^{n}\right)$ играют важную роль в теории операторов Шрёдингера. Там же, в [2], были получены эффективные достаточные условия принадлежности функций таким пространствам мультипликаторов. В этой работе мы получим полное описание пространства мультипликаторов из $H_{p}^{\alpha}\left(\mathbb{R}^{n}\right)$ в $H_{p^{\prime}}^{-\alpha}\left(\mathbb{R}^{n}\right)$ (мы обозначаем его через $M_{p}^{\alpha}$ ) при условии $\alpha>n / p$. В случае $\alpha \leqslant p / n$ мы получим теоремы вложения соболевских пространств с негативными индексами гладкости в пространства $M_{p}^{\alpha}$ (достаточные условия принадлежности к $M_{p}^{\alpha}$ ).

Напомним, что пространства Соболева (или бесселевых потенциалов) $H_{p}^{\alpha}:=H_{p}^{\alpha}\left(\mathbb{R}^{n}\right)$ определяются как замыкание пространства основных функций $D$ по норме

$$
\|u\|_{\alpha, p}:=\left\|\Lambda^{\alpha} u\right\|_{L_{p}}
$$

где $\Lambda^{\alpha}=F^{-1}\left[\left(1+|\xi|^{2}\right)^{\alpha / 2} F\right]$, а $F$ - преобразование $\Phi$ урье в $\mathbb{R}^{n}$.

Введем обозначение

$$
H_{p, \text { loc }}^{\alpha}\left(\mathbb{R}^{n}\right)=\left\{v \in D^{\prime}: v(x) \varphi(x) \in H_{p}^{\alpha} \quad \forall \varphi \in D\right\}
$$

Работа первого автора выполнена при поддержке фонда KOSEF (Korea Science and Engineering Foundation), грант № 1999-2-102-003-5. Работа второго автора выполнена при поддержке Российского фонда фундаментальных исследований, грант № 01-01-00691. 
где $D^{\prime}$ - пространство распределений на $D$. Функцию $\mu(x) \in H_{p^{\prime}, \text { lос }}^{-\alpha}$ назовем мультuпликатором из $H_{p}^{\alpha}$ в $H_{p^{\prime}}^{-\alpha}$, если

$$
\|\mu(x) \varphi(x)\|_{-\alpha, p^{\prime}} \leqslant C\|\varphi\|_{\alpha, p} \quad \forall \varphi \in D,
$$

где через $C$ обозначается (здесь и в дальнейшем) константа, не зависящая от $\varphi \in D$.

В этом определении предполагается, что $\varphi \in D$ (вместо $\varphi \in H_{p}^{\alpha}$ ), поскольку при $\mu(x) \in H_{p^{\prime}, \text { loc }}^{-\alpha}$ произведение $\mu(x) \varphi(x)$ определено в $D^{\prime}$ лишь для бесконечно гладких $\varphi$. Однако $D$ плотно в $H_{p}^{\alpha}$, поэтому из оценки $(1)$ для $\varphi \in D$ следует, что оператор умножения на функцию $\mu(x)$ может быть продолжен на все $H_{p}^{\alpha}$ как ограниченньй оператор из $H_{p}^{\alpha}$ в $H_{p^{\prime}}^{-\alpha}$. Очевидно, функции $\mu(x)$, удовлетворяющие неравенству $(1)$, образуют банахово пространство, которое мы обозначим через $M\left[H_{p}^{\alpha} \rightarrow H_{p^{\prime}}^{-\alpha}\right]$, или более кратко $M_{p}^{\alpha}$. Норма в этом пространстве определяется как

$$
\|\mu\|_{M_{p}^{\alpha}}=\inf C
$$

где нижняя грань берется по всем константам $C$, для которых выполнено неравенство (1).

Вначале зададимся вопросом: для каких индексов $\gamma \leqslant \alpha, r \geqslant 1$ имеет место непрерьвное вложение с оценкой нормы

$$
H_{r}^{-\gamma} \in M\left[H_{p}^{\alpha} \rightarrow H_{p^{\prime}}^{-\alpha}\right], \quad\|\mu\|_{M_{p}^{\alpha}} \leqslant C\|\mu\|_{-\gamma, r} ?
$$

Ответ на этот вопрос дает следующая теорема.

ТЕорема 1. Вложение (2) с оценкой норм имеет место тогда и только тогда, когда выполняется мультипликативная оценка

$$
\|\varphi(x) \psi(x)\|_{\gamma, r^{\prime}} \leqslant C\|\varphi\|_{\alpha, p}\|\psi\|_{\alpha, p} \quad \forall \varphi, \psi \in D,
$$

где число $1 \leqslant r^{\prime} \leqslant \infty$ определяется из условия сопряжения $1 / r+1 / r^{\prime}=1$.

ДокАЗАТЕЛЬСТво. Достаточность. Пусть $\mu(x) \in H_{r}^{-\gamma}$ и вьполнена оценка (3). Тогда для всех $\varphi, \psi \in D$ имеем

$$
\begin{aligned}
\|\mu \varphi\|_{-\alpha, p^{\prime}} & =\sup _{0 \neq \psi \in H_{p}^{\alpha}} \frac{|(\mu \varphi, \psi)|}{\|\psi\|_{\alpha, p}}=\sup _{0 \neq \psi \in H_{p}^{\alpha}} \frac{|(\mu, \bar{\varphi} \psi)|}{\|\psi\|_{\alpha, p}} \\
& \leqslant \sup _{0 \neq \psi \in H_{p}^{\alpha}} \frac{\|\mu\|_{-\gamma, r}\|\bar{\varphi} \psi\|_{\gamma, r^{\prime}}}{\|\psi\|_{\alpha, p}} \leqslant C\|\mu\|_{-\gamma, r}\|\varphi\|_{\alpha, p},
\end{aligned}
$$

где константа $C$ та же, что и в (3). По определению получаем $\mu \in M_{p}^{\alpha}$ и $\|\mu\|_{M_{p}^{\alpha}} \leqslant$ $C\|\mu\|_{-\gamma, r}$.

Необходимость. Пусть справедливо вложение (2) с оценкой норм. Тогда для $\varphi, \psi \in D$ выполнены неравенства

$$
\begin{aligned}
\|\varphi \psi\|_{\gamma, r^{\prime}} & =\sup _{f \in H_{r}^{-\gamma}} \frac{|(f, \varphi \psi)|}{\|f\|_{-\gamma, r}} \leqslant \sup _{\mu \in M_{p}^{\alpha}} \frac{|(\mu \bar{\varphi}, \psi)|}{C^{-1}\|\mu\|_{M_{p}^{\alpha}}} \\
& \leqslant \sup _{\mu \in M_{p}^{\alpha}} \frac{\|\mu \bar{\varphi}\|_{-\alpha, p^{\prime}}\|\psi\|_{\alpha, p}}{C^{-1}\|\mu\|_{M_{p}^{\alpha}}} \leqslant C\|\varphi\|_{\alpha, p}\|\psi\|_{\alpha, p}
\end{aligned}
$$


причем для получения последнего неравенства мы использовали оценку (1) с $C=$ $\|\mu\|_{M_{p}^{\alpha}}$. Следовательно, вьполнена оценка (2), и доказательство завершено.

Выберем неотрицательную функцию $\eta(x) \in D$ такую, что $\eta(x) \equiv 1$ при $|x| \leqslant 1$. Вве-

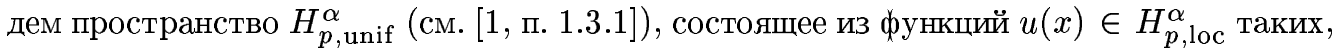
что

$$
\|u\|_{\alpha, p, \text { unif }}:=\sup _{z \in \mathbb{R}^{n}}\|\eta(x-z) u(x)\|_{\alpha, p}<\infty .
$$

Норма в пространстве $H_{p \text {, unif }}^{\alpha}$ зависит от выбора неотрищательной пробной функции $\eta(x)$, но для всех таких функций соответствуюшие нормы эквивалентны.

Если $\mu(x) \in M_{p}^{\alpha}$, то и $\eta(x) \mu(x) \in M_{p}^{\alpha}$. Введем новую норму в $M_{p}^{\alpha}$ :

$$
\|\mu \mu\|_{M_{p}^{\alpha}}=\sup _{z \in \mathbb{R}^{n}}\|\eta(x-z) \mu(x)\|_{M_{p}^{\alpha}} .
$$

Приведенная ниже теорема известна для мультипликаторов в соболевских пространствах с неотрицательными индексами (см. [1, п. 2.1.3]). Здесь мы предлагаем независимое доказательство применительно к нашему случаю.

ТЕОРема 2. Нормы $\|\mid \cdot\| u\|\cdot\|$ в $M_{p}^{\alpha}$ әквивалентны.

ДокАЗАТЕЛЬСтво. Неравенство

$$
\|\mu \mid\| M_{p}^{\alpha} \leqslant C\|\mu\|_{M_{p}^{\alpha}}
$$

следует из определения. Для доказательства обратной оценки нам потребуется известная лемма о разбиении единицы (см., например, $[3, \S 1.4])$. Согласно этой лемме существует набор функций $\left\{\chi_{j}(x)\right\}_{j=1}^{\infty}$, удовлетворяющих следующим условиям:

(i) $\chi_{j}(x) \in D, 0 \leqslant \chi_{j}(x) \leqslant 1$ и $\sum_{j=1}^{\infty} \chi_{j}(x) \equiv 1$;

(ii) носители функций $\chi_{j}$ содержатся в шарах радиуса 1 (с различньми центрами), при этом любая фиксированная точка $x \in \mathbb{R}^{n}$ покрьвается носителями не более чем $N$ функций (т.е. $\chi_{j}(x) \neq 0$ не более чем для $N$ функций и $N$ не зависит от $x$ );

(iii) $\left|D^{\ell} \chi_{j}(x)\right| \leqslant C$, где $C$ зависит лиш от мультииндекса $\ell=\left(\ell_{1}, \ldots, \ell_{n}\right)$.

Пусть семейство $\left\{\chi_{j}\right\}_{1}^{\infty}$ обладает этими свойствами. Тогда

$$
\sum_{j=1}^{\infty}\left\|\varphi \chi_{j}\right\|_{\alpha, p} \leqslant C\|\varphi\|_{\alpha, p}, \quad \varphi \in D
$$

где $C$ зависит только от $\alpha, p$ и от выбора $\left\{\chi_{j}\right\}_{1}^{\infty}$. Поскольку при всех $j$ носитель $\chi_{j}$ содержится в шаре радиуса 1 , а $\eta(x)=1$ при $|x| \leqslant 1$, то существуют $z_{j} \in \mathbb{R}^{n}$ такие, что $\chi_{j}(x)=\eta\left(x-z_{j}\right) \chi_{j}(x)$. Обозначая $\varphi_{j}(x)=\chi_{j}(x) \varphi(x)$ для $\varphi \in D$, получаем $\|\mu \varphi\|_{-\alpha, p^{\prime}}=\left\|\sum_{j=1}^{\infty} \eta\left(x-z_{j}\right) \mu(x) \varphi_{j}(x)\right\|_{-\alpha, p^{\prime}} \leqslant\|\mu\|\left\|_{M_{p}^{\alpha}} \sum_{j=1}^{\infty}\right\| \varphi_{j}\left\|_{\alpha, p} \leqslant C\right\| \mu\|\|_{M_{p}^{\alpha}}\|\varphi\|_{\alpha, p}$. Теперь из определения следует, что

$$
\|\mu\|_{M_{p}^{\alpha}} \leqslant C\|\| \mu \|_{\alpha, p}
$$

Теорема доказана. 
ТЕОремА 3. Верны следующие утверждения:

1) для всех $\alpha \geqslant 0$ и $p \geqslant 1$ справедливы вложения с оченками норм

$$
M_{p}^{\alpha} \subset H_{p^{\prime}, \text { unif }}^{-\alpha}, \quad\|\mu\|_{-\alpha, p^{\prime}, \text { unif }} \leqslant C\|\mu\|_{M_{p}^{\alpha}} ;
$$

2) вложение с оценкой норм

$$
H_{r, \text { unif }}^{-\gamma} \subset M_{p}^{\alpha}, \quad\|\mu\|_{M_{p}^{\alpha}} \leqslant C\|\mu\|_{-\gamma, r, \text { unif }}
$$

справедливо тогда и только тогда, когда выполняется неравенство (3).

ДокАЗАтЕльство. Первое утверждение вытекает из определения. Действительно, если $\mu(x) \in M_{p}^{\alpha}$ и $\eta(x)$ - гладкая срезающая функция, то

$$
\|\mu(x) \eta(x-z)\|_{-\alpha, p^{\prime}} \leqslant\|\mu\|_{M_{p}^{\alpha}}\|\eta(x-z)\|_{\alpha, p}=\|\mu\|_{M_{p}^{\alpha}}\|\eta(x)\|_{\alpha, p}=C\|\mu\|_{M_{p}^{\alpha}},
$$

где $C=\|\eta(x)\|_{\alpha, p}$ не зависит от $z$. Поэтому $\mu \in H_{p^{\prime}, \text { unif }}^{-\alpha}$ и верна оценка (4).

Второе утверждение является следствием теорем 1 и 2. Очевидно, пространство $H_{r}^{-\gamma}$ непрерьвно вложено в $H_{r, \text { unif }}^{-\gamma}$. Поэтому согласно теореме 1 оценка (5) влечет (3). Обратно, если вьполнена оценка (3) и $\mu \in H_{r, \mathrm{unif}}^{-\gamma}$, то $\mu(x) \eta(x-z) \in H_{r}^{-\gamma} \subset M_{p}^{\alpha}$ и

$$
\|\mu(x) \eta(x-z)\|_{M_{p}^{\alpha}} \leqslant C\|\mu(x) \eta(x-z)\|_{-\gamma, r} .
$$

Таким образом, $\|\mu \mu \mid\|_{M_{p}^{\alpha}} \leqslant C\|\mu\|_{-\gamma, r, \text { unif }}$ и (5) вытекает из теоремы 2. Теорема доказана.

Согласно теореме Стрихардса (см. [4] или [1, п. 2.2.9]) пространство мультипликаторов $M\left[H_{p}^{\alpha} \rightarrow H_{p}^{\alpha}\right]$ совпадает с $H_{p \text {, unif }}^{\alpha}$, если выполено условие $\alpha>n / p$. Полученное ниже утверждение можно считать аналогом этой теоремы для мультипликаторов в дуальных пространствах.

Tеорема 4. Пусть $\alpha>n / p$. Тогда пространства $M\left[H_{p}^{\alpha} \rightarrow H_{p^{\prime}}^{-\alpha}\right] u H_{p^{\prime}}^{-\alpha}$,unif совпадают и нормы в этих пространствах әквивалентны.

ДокАЗАТЕЛЬСтво. Согласно первому утверждению теоремы 3 вложение $M_{p}^{\alpha} \subset$ $H_{p^{\prime}, \text { unif }}^{-\alpha}$ с оценкой норм верно для всех $\alpha \geqslant 0$ и $p \geqslant 1$. Чтобы доказать обратное включение, в соответствии со вторьм утверждением теоремы 3 достаточно проверить неравенство (3) с $\gamma=\alpha$ и $r^{\prime}=p$. Но для этих значений индексов оценка (3) следует из того факта, что $H_{p}^{\alpha}$ является алгеброй при $\alpha>n / p$ (см. $\left.[1, \S 1.7]\right)$. Теорема доказана.

В случае $\alpha \leqslant n / p$ дать описание пространства $M_{p}^{\alpha}$ в терминах пространств Соболева уже невозможно. По аналогии с теорией мультипликаторов в соболевских пространствах с неотрицательными индексами гладкости такое описание можно провести в терминах емкости, хотя это требует дополнительной серьезной работы (см. результаты Вербицкого в [1]). Однако проверка условий в терминах емкостей трудно осуществима, поэтому полезно иметь эффективные достаточные условия принадлежности функций рассматриваемым пространствам мультипликаторов. Нам удалось найти точные условия на индексы $\gamma$ и $r$, при которых справедливо вложение $H_{r, \text { unif }}^{-\gamma} \subset M_{p}^{\alpha}$ вместе с оценкой норм. Здесь мы приведем две теоремы такого типа. Результат первой из них важен лишь при $\alpha=n / p$ поскольку при $\alpha<n / p$, более точное утверждение дает вторая из этих теорем. 
Теорема 5. Пусть $p>1 u \max \{0,(2-p) n / p\}<\alpha \leqslant n / p$. Тогда справедливо вложение $H_{r, \text { unif }}^{-\gamma} \subset M_{p}^{\alpha}$ вместе с оченкой норм, если числа $\gamma$ и $r$ подчинень условиям

$$
-2\left(\frac{n}{p}-\alpha\right) \leqslant \gamma \leqslant \alpha \quad u \quad r>\frac{p n}{p(n+2 \alpha-\gamma)-2 n} .
$$

ДоказАТЕЛЬСтво. Достаточно доказать это утверждение при $\gamma=\alpha$. Действительно, предположим, что доказано вложение $H_{r_{1}}^{\alpha} \subset M_{p}^{\alpha}$ вместе с оценкой норм при $r_{1}>p n /(p(n+\alpha)-2 n)$. В силу теоремы Соболева $H_{r}^{\gamma} \subset H_{r_{1}}^{\alpha}$, если $1 / r-1 / r_{1}=\alpha-\gamma \geqslant 0$. Следовательно, $H_{r}^{\gamma} \subset M_{p}^{\alpha}$, если $r=r_{1} /\left(1+r_{1}(\alpha-\gamma)\right)$, т.е. если $r>p n /(p(n+2 \alpha-\gamma)-2 n)$. Здесь число $\gamma(\gamma \leqslant \alpha)$ можно выбрать любым, лишь бы выполнялось условие $r>1$. Это приводит к условию $\gamma>-2(n / p-\alpha)$.

Итак, пусть $\gamma=\alpha$. Положим $\nu=n / p+\varepsilon, \varepsilon>0$, и запишем следуюшие неравенства:

$$
\|\varphi \psi\|_{\nu, p} \leqslant C\|\varphi\|_{\nu, p}\|\psi\|_{\nu, p}, \quad\|\varphi \psi\|_{0, p / 2} \leqslant\|\varphi\|_{0, p}\|\psi\|_{0, p}
$$

Первое из неравенств следует из того, что $H_{p}^{\nu}$ при $\nu>n / p$ есть алгебра (теорема Стрихардса), а второе есть обычное неравенство Гёльдера. Теперь используя полилинейную интероляционную теорему (см., например, $[5, \S 4.4])$, мы приходим к неравенству

$$
\|\varphi \psi\|_{\alpha, s} \leqslant C\|\varphi\|_{\alpha, p}\|\psi\|_{\alpha, p}
$$

для

$$
0 \leqslant \alpha \leqslant \nu, \quad s=\frac{p(n+\varepsilon)}{2 n-\alpha p+2 p \varepsilon}, \quad s^{\prime}=\frac{p(n+\varepsilon)}{p n+p \alpha-2 n-p \varepsilon} .
$$

Здесь мы обрашаем внимание, что в случае $1 \leqslant p<2$ в пространствах $H_{p / 2}^{0}=L_{p / 2}$ нет обычной нормы, их можно оснастить лишь квазинормой. Однако использованная нами интерполяционная теорема справедлива и для квазинормированных пространств (см. [5, §3.10]). По условию теоремы $(2-p) n / p<\alpha \leqslant n / p$, что влечет $s, s^{\prime}>1$ при достаточно малом $\varepsilon>0$. Для завершения доказательства надо выбрать $\varepsilon$ таким, чтобы $s^{\prime}=r$, а затем воспользоваться теоремами 1 и 3.

В случае $\alpha=n / p$ результат нельзя улучшить, заменив неравенство для $r$ на соответствуюшее равенство (т.е. положить $\varepsilon=0$ ). Однако это можно сделать при $\alpha<n / p$. Доказательство этого факта требует более глубокого анализа. Отметим, что при целом $\alpha$ доказательство можно провести значительно проще (см. [2]).

Tеорема 6. Пусть $p>1 u \max \{0,(2-p) n / p\}<\alpha<n / p$. Тогда справедливо вложение $H_{r, \text { unif }}^{-\gamma} \subset M_{p}^{\alpha}$ вместе с оченкой норм, если числа $\gamma, r$ подчинены условиям

$$
-2\left(\frac{n}{p}-\alpha\right) \leqslant \gamma \leqslant \alpha \quad u \quad r \geqslant \frac{p n}{p(n+2 \alpha-\gamma)-2 n} .
$$


ДокАЗАТЕЛЬСТво. Как и в предыдущей теореме, можно ограничиться случаем $\gamma=\alpha$, причем утверждение нужно доказать только для $r=p n /(p(n+\alpha)-2 n)$. Согласно теореме 3 нужно доказать оценку

$$
\|\varphi \psi\|_{\alpha, s} \leqslant C\|\varphi\|_{\alpha, p}\|\psi\|_{\alpha, p} \quad \forall \varphi, \psi \in D
$$

где $s=p n /(2 n-\alpha p)>1$. Для доказательства этой оценки мы будем использовать методы, развитые в статье Полкинга [6].

Рассмотрим нелинейный оператор

$$
D_{\rho, q}^{\eta} u(x)=\int_{0}^{\infty}\left(\int_{B_{1}}|u(x+\xi y)-u(x)|^{\rho} d y\right)^{q / \rho} \xi^{-1-\eta q} d \xi
$$

где $\eta>0, \rho \geqslant 1, q \geqslant 2$. Нам потребуются следующие утверждения, доказанные в работах Полкинга [6] и Стрихардса [4].

ПРЕДЛОЖЕНИЕ А (см. [6]). Пусть $0<\gamma<1,1 \leqslant \rho \leqslant q, 2 \leqslant q<\infty, r>\max \{1$, $n \rho /(n+\gamma \rho)\}$. Тогда выполнено следующее неравенство:

$$
\left\|D_{\rho, q}^{\eta} u(x)\right\|_{0, r} \leqslant C\|u(x)\|_{\eta, r}, \quad u \in H_{r}^{\eta}
$$

әде константа $C$ зависит от $\eta, \rho, q, r, n$, но не зависит от $u(x)$.

ПРЕДЛОЖЕНИЕ В (см. [6]). Пусть $1 / \rho_{1}+1 / \rho_{2}=1 / \rho, 1 / q_{1}+1 / q_{2}=1 / q u \eta_{1}+\eta_{2}=\eta$.

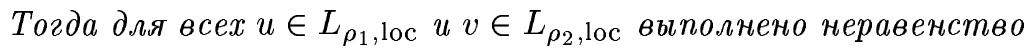

$$
\left|D_{\rho, q}^{\eta}(u v)(x)\right| \leqslant\left|u(x) D_{\rho, q}^{\eta} v(x)\right|+\left|v(x) D_{\rho, q}^{\eta} u(x)\right|+\left|D_{\rho_{1}, q_{1}}^{\eta_{1}} u(x) D_{\rho_{2}, q_{2}}^{\eta_{2}} v(x)\right| \text {. }
$$

ПРЕДЛОЖЕНИЕ С (см. [6], [4]). Имеет место следующее соотношение:

$$
\|v\|_{\eta, p} \asymp\left\|D_{1,2}^{\eta} v\right\|_{0, p}+\|v\|_{0, p}, \quad p \geqslant 1, \eta>0, \quad v(x) \in H_{p}^{\eta},
$$

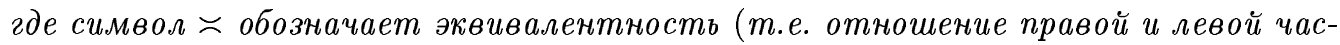
тей ограничено с обеих сторон константами, не зависящими от $v(x))$.

Используя эти утверждения, докажем оценку (6) для $s=p n /(2 n-\alpha p)$. Положим $\alpha=[\alpha]+\eta$, где $[\alpha]$ обозначает целую часть $\alpha$ и $0 \leqslant \eta<1$. Будем предполагать, что $\eta>0$ (для $\eta=0$ доказательство проще и может быть получено при помощи обычной формулы Лейбница). Хорошо известно следующее соотношение (см., например, [7]):

$$
\|\varphi \psi\|_{\alpha, s} \asymp \sum_{|\ell| \leqslant[\alpha]}\left\|D^{\ell}(\varphi \psi)\right\|_{\eta, s}
$$

Согласно предложению С правая часть оценивается сверху величиной (в дальнейшем в оценках мы опускаем константы)

$$
\sum_{|j|+|m| \leqslant[\alpha]}\left(\left\|D_{1,2}^{\eta}\left(D^{j} \varphi D^{m} \psi\right)\right\|_{0, s}+\left\|D^{j} \varphi D^{m} \psi\right\|_{0, s}\right) .
$$


Это выражение может быть оценено сверху с учетом предложения В следующей величиной (мы будем оценивать только первое слагаемое, второе оценивается существенно проще):

$$
\begin{gathered}
\sum_{|j|+|m| \leqslant[\alpha]}\left(\left\|D^{m} \psi D_{1,2}^{\eta}\left(D^{j} \varphi\right)\right\|_{0, s}+\left\|D^{j} \varphi D_{1,2}^{\eta}\left(D^{m} \psi\right)\right\|_{0, s}\right. \\
\left.+\left\|\left(D_{\rho_{1}, q_{1}}^{\eta_{1}} D^{j} \varphi\right)\left(D_{\rho_{2}, q_{2}}^{\eta_{2}} D^{m} \psi\right)\right\|_{0, s}\right) .
\end{gathered}
$$

Теперь выберем подходящим образом числа $\eta_{1}, \rho_{1}, q_{1}$. Положим

$$
\begin{gathered}
0<\eta_{1}<\max \left\{\eta, \frac{n}{p}-\alpha\right\}, \quad \eta_{2}=\eta-\eta_{1}, \\
\rho_{1}=\frac{n}{|j|+\eta_{1}}, \quad \rho_{2}=\frac{n}{n-|j|-\eta_{1}}, \quad q_{1}=2 \rho_{1}, \quad q_{2}=2 \rho_{2} .
\end{gathered}
$$

Так как $|j|+\eta_{1}<\alpha$, то $\rho_{1}, \rho_{2}>1$ и $1 / \rho_{1}+1 / \rho_{2}=1$.

Оценим третье слагаемое в (7). Положим

$$
r=\frac{2 n-\alpha p}{n+\left(|j|+\eta_{1}-\alpha\right) p}, \quad r^{\prime}=\frac{2 n-\alpha p}{n-\left(|j|+\eta_{1}\right) p} .
$$

Очевидно, $r, r^{\prime}>0,1 / r+1 / r^{\prime}=1$, поэтому $r, r^{\prime}>1$. С помощью неравенства Гёльдера третье слагаемое в (7) оценивается выражением

$$
\left(\left\|D_{\rho_{1}, 2 \rho_{1}}^{\eta_{1}} D^{j} \varphi\right\|_{0, r s}\right)\left(\left\|D_{\rho_{2}, 2 \rho_{2}}^{\eta_{2}} D^{m} \psi\right\|_{0, r^{\prime} s}\right) .
$$

Согласно предложению А последнее выражение можно оценить величиной

$$
\left\|D^{j} \varphi\right\|_{\eta_{1}, r s}\left\|D^{m} \psi\right\|_{\eta_{2}, r^{\prime} s}
$$

если только выполненены условия

$$
r s>1, \quad r s>\frac{n \rho_{1}}{n+\eta_{1} \rho_{1}}, \quad r^{\prime} s>1, \quad r^{\prime} s>\frac{n \rho_{2}}{n+\eta_{2} \rho_{2}} .
$$

Проверим справедливость этих условий. Используя (8) и (9), получаем

$$
r s=\frac{p n}{n-p\left(\alpha-|j|-\eta_{1}\right)}>\frac{p n}{n}=p>1,
$$

поскольку $\alpha>|j|+\eta_{1}$;

$$
\begin{gathered}
r s=\frac{n}{(n / p-\alpha)+|j|+\eta_{1}}>\frac{n}{|j|+2 \eta_{1}}=\frac{n}{n / \rho_{1}+\eta_{1}}=\frac{n \rho_{1}}{n+\eta_{1} \rho_{1}} \\
r^{\prime} s=\frac{n \rho}{n-\left(|j|+\eta_{1}\right) p}>p>1 ; \\
r^{\prime} s=\frac{n}{n / \rho-|j|-\eta_{1}}>\frac{n}{r / \rho-|j|-\eta_{1}+(n-n / p)}=\frac{n}{n / \rho_{2}+\eta_{2}}=\frac{n \rho_{2}}{n+\eta_{2} \rho_{2}} .
\end{gathered}
$$


Далее, выражение (10) оценивается величиной

$$
\|\varphi\|_{|j|+\eta_{1}, r s}\|\psi\|_{|m|+\eta_{2}, r^{\prime} s} \leqslant C\left\|_{\varphi}\right\|_{\alpha, p}\|\psi\|_{\alpha, p}
$$

Последняя оценка получается из теоремы вложения Соболева с учетом неравенств

$$
|j|+\eta_{1}-\frac{n}{p} \leqslant \alpha-\frac{n}{r s}, \quad|m|+\eta_{2}-\frac{n}{p} \leqslant \alpha-\frac{n}{r^{\prime} s} .
$$

Первое из этих неравенств следует из (11) и условия $|j|+\eta_{1}<\alpha$, а второе - из (12) и условия $|m|+\eta_{2}<\alpha$.

Оценки первого и второго слагаемого в (7) могут быть получены таким же образом, полагая $\eta_{1}=\eta, \eta_{2}=0$ для первого слагаемого и $\eta_{1}=0, \eta_{2}=\eta$ для второго. Теорема доказана.

Применение полученных результатов к эллипическим операторам общего вида мы оставляем за пределами этой работы. Здесь дадим лишь достаточное условие на потенциал-распределение $q(x)$, гарантирующее корректность определения соответствующего оператора Шрёдингера

$$
\mathscr{L} u(x)=(-\Delta+q(x)) u(x)
$$

в пространстве $L_{2}\left(\mathbb{R}^{n}\right)$. Этот результат дополняет полученные ранее результаты [2] и [8].

Теорема 7. Пусть $\eta(x)$ - гладкая, неотрицательная функция с компактным носителем в $\mathbb{R}^{n}$, не обращающаяся тождественно в нуль. Пусть функиия-распределение $q(x)$ удовлетворяет условию:

$$
\begin{array}{ll}
\lim _{|z| \rightarrow \infty}\|q(x) \eta(x-z)\|_{-1,2}=0, & \text { если } n=1 ; \\
\lim _{|z| \rightarrow \infty}\|q(x) \eta(x-z)\|_{-1,2+\varepsilon}=0 & \text { при некотором } \varepsilon>0, \text { если } n=2 ; \\
\lim _{|z| \rightarrow \infty}\|q(x) \eta(x-z)\|_{-1, n}=0, & \text { если } n \geqslant 3 .
\end{array}
$$

Тогда оператор (13) корректно определен как оператор в пространстве $H_{2}^{-1}\left(\mathbb{R}^{n}\right)$ с областью определения $H_{2}^{1}\left(\mathbb{R}^{n}\right)$, а его резольвентное мнохество непусто. Более того, спектр $\mathscr{L}$ лежит в произвольно малом секторе $\left|\arg \left(\lambda+\lambda_{0}\right)\right|<\varepsilon$, если $\lambda_{0}=\lambda_{0}(\varepsilon)$ достаточно большое число. Если функиия $q(x)$ вещественна, то $\mathscr{L}$ является самосопряженным оператором в пространстве $H_{2}^{-1}\left(\mathbb{R}^{n}\right)$ с областью определения $\mathscr{D}(\mathscr{L})=H_{2}^{-1}\left(\mathbb{R}^{n}\right)$. Сужение оператора $\mathscr{L}$ на область

$$
\mathscr{D}(\mathscr{L})=\left\{u(x) \in H_{2}^{1} \mid-\Delta u(x)+q(x) u(x) \in L_{2}\left(\mathbb{R}^{n}\right)\right\}
$$

является самосопряженным полуограниченным оператором в $L_{2}$, причем область определения квадратного корня $(\mathscr{L}+\mu)^{1 / 2}($ при достаточно больиих $\mu)$ cовпадает с $H_{2}^{1}\left(\mathbb{R}^{n}\right)$. 
ДоказАтельство. Рассмотрим, например, случай $n=1$ (ср. [2], [8]). Условие теоремы влечет возможность представления $q(x)=q_{0}(x)+q_{\varepsilon}(x)$, где $q_{0}(x)$ - функция с финитным носителем в пространстве $H_{2}^{-1}$, a $\left\|q_{\varepsilon}(x)\right\|_{-1,2, \text { unif }}<\varepsilon$. Тогда согласно теореме 3 норма функции $q_{\varepsilon}(x)$ в пространстве $M_{2}^{1}$ не превьшыет величины $C \varepsilon$. С учетом теоремы 4 легко заметить, что оператор умножения на $q_{0}(x)$ явяется компактным мультипликатором в $M_{2}^{1}$, а потому из указанного представления имеем оценку

$$
\|q(x) u(x)\|_{-1,2} \leqslant \varepsilon_{1}\|u(x)\|_{1,2}+C\|u(x)\|_{0,2},
$$

где число $\varepsilon_{1}>0$ можно выбрать произвольно малым, в частности, меньшим 1 . Теперь оператор $\mathscr{L}$ можно корректно определить в $H_{2}^{-1}\left(\mathbb{R}^{n}\right)$ методом квадратичных форм, причем все другие утверждения теоремы получаются с помощью средств этого метода (см. подробности в [2]).

Результаты этой заметки сообщались авторами в Postech и Seoul National университетах (январь 2001 года), на семинарах в Московском университете (февраль 2001 года) и на конференции по теории операторов в Центре Банаха (Варшава, июль-август 2001 года).

\section{СПИСОК ЦИТИРОВАННОЙ ЛИТЕРАТУРЫ}

[1] Мазья В. Г., Шапошникова Т. О. Теория мультипликаторов в пространствах дифференцируемых функций: Изд-во Ленинградского ун-та, 1986.

[2] Нейман-заде М. И., Шкаликов А. А. Операторы Шрёдингера с сингулярынми потенциалами из пространств мультипликаторов // Матем. заметки. 1999. Т. 66. № 4. С. 599-609.

[3] Хермандер Л. Анализ линейных дифференциальных операторов с частными производными. Т.1: Теория распределений и Фурье анализ.. М.: Мир, 1990.

[4] Strichartz R.S. Multipliers in fractional Sobolev spaces // J. Math. Mech. 1967. V. 16. P. 1031-1060.

[5] Bergh J., Löfström J. Interpolation Spaces // Grund. der math. Wiss. V. 223. Berlin: Springer Verlag, 1976.

[6] Polking J. C. A Leibniz formula for some differential operators of fractional order // Indiana Univ. Math. J. 1972. V. 27. P. 1019-1029.

[7] Stein E. M. Singular Integrals and Differentiability Properties of Functions. Princeton: Princeton Univ. Press, 1970.

[8] Савчук А. М., Шкаликов А. А. Операторы Штурма-Лиувилля с сингулярными потенциалами // Матем. заметки. 1999. Т. 64. №6. С. 897-912.

(Дж.-Г. Бак) Postech University in South Korea

Поступило (А. А. Шкаликов) Московский государственный университет им. М. В. Ломоносова 30.10 .2001

E-mail: bak@postech.ac.kr, shkal@mech.math.msu.su 